\title{
Earle Slices Associated with Involutions for Once Punctured Torus
}

\author{
$\mathrm{Na}$ LI \\ Nagoya University \\ (Communicated by K. Matsuzaki)
}

\begin{abstract}
In this paper, we will study Earle slices of quasi-fuchsian space for once punctured torus associated with involutions of its fundamental group induced by orientation reversing diffeomorphism of this surface. First we classify Earle slices into two types: rhombic Earle slices and rectangular Earle slices. The main purpose of this paper is to study the configuration of Earle slices. Especially, we obtain a necessary and sufficient condition for two Earle slices to intersect each other. We also show that the union of all Earle slices is connected. In the end, we describe Earle slices by using trace coordinates of quasi-fuchsian space.
\end{abstract}

\section{Introduction}

Earle slices, which are holomorphic slices of quasi-fuchsian spaces, were first introduced by Earle [2] in 1983. Actually Earle considered in [2] the case of closed Riemann surface of genus $\geq 2$. Later Komori and Series [6] studied the case of once punctured torus. In this paper, we consider all of the Earle slices for once punctured torus $S$ associated with involutions of the fundamental group $\pi_{1}(S)$ induced by orientation reversing diffeomorphism of $S$. We will classify these Earle slices into two types: rhombic Earle slices and rectangular Earle slices. Here rhombic Earle slices are associated with involutions exchanging some pair $(\alpha, \beta)$ of generators of $\pi_{1}(S)$, while rectangular Earle slices are associated with involutions which take $\alpha$ to $\alpha$ and $\beta$ to $\beta^{-1}$ for some pair $(\alpha, \beta)$ of generators of $\pi_{1}(S)$. Komori and Series [6] studied rhombic Earle slices, and Komori [7] studied rectangular Earle slices.

In this paper, we study the configuration of Earle slices in $\mathcal{Q F}(S)$ of once punctured torus. The paper is organized as follows. In Section 2, we set up notations and recall some definitions we will use later. In Section 3, we give the classification of Earle slices. In Section 4 , we consider the action of mapping class group on the quasi-fuchsian space and determine the stabilizer subgroup of each Earle slice.

The main results appear in Section 5. We give a necessary and sufficient condition for two Earle slices to intersect each other. More precisely, we first obtain a necessary and sufficient condition for two rhombic Earle slices to intersect each other. We next show that two 
rectangular Earle slices do not intersect. Finally we obtain a necessary and sufficient condition for one rhombic Earle slice and one rectangular Earle slice to intersect each other.

Besides, we show that for any Earle slice, there exists a unique Earle slice of different kind that intersects it. We also show that for any rhombic Earle slice, there exist exactly four distinct rhombic Earle slices that intersect it. In the end of Section 5, we show that the union of all rhombic Earle slices is connected. As a consequence, the union of all Earle slices is also connected.

Finally in Section 6, we describe Earle slices using trace coordinates of the quasifuchsian space.

\section{Preliminary}

2.1. Teichmüller space. Let $S$ be an oriented once punctured torus and let $\pi_{1}(S)$ be its fundamental group. An ordered pair $(\alpha, \beta)$ of generators of $\pi_{1}(S)$ is called canonical if the algebraic intersection number $i(\alpha, \beta)$ is equal to +1 with respect to the given orientation of $S$. The Teichmüller space $\mathcal{T}(S)$ is defined to be the set of equivalence classes of $(X, f)$, where $X$ is a hyperbolic Riemann surface of finite hyperbolic area and $f: \pi_{1}(S) \rightarrow \pi_{1}(X)$ is a typepreserving isomorphism which is induced by orientation preserving homeomorphism from $S$ to $X$. Here type-preserving means $f$ maps the homotopy class of loops around the puncture of $S$ to the same homotopy class of loops around the puncture of $X$. $(X, f)$ is equivalent to $(Y, g)$ if and only if there exists a conformal map $h$ from $X$ to $Y$ that induces an isomorphism $h_{*}: \pi_{1}(X) \rightarrow \pi_{1}(Y)$ such that $g^{-1} h_{*} f$ is an inner automorphism of $\pi_{1}(S)$. We denote the equivalence class of $(X, f)$ by $[X, f]$. For short, let $X$ denote $[X, f] \in \mathcal{T}(S)$.

By fixing a pair $(\alpha, \beta)$ of canonical generators of $\pi_{1}(S)$, the Teichmüller space $\mathcal{T}(S)$ can be naturally identified with the upper half plane $\mathbb{H}=\{z \in \mathbb{C} \mid \Im z>0\}$ as follows: for any point $\tau \in \mathbb{H}$, we associate it with the point $[X, f] \in \mathcal{T}(S)$ where $X$ is equal to $\mathbb{C} /(\mathbb{Z} \oplus \mathbb{Z} \tau)$ with one point removed, and $f: \pi_{1}(S) \rightarrow \pi_{1}(X)$ is a type-preserving isomorphism that sends $\alpha$ and $\beta$ to the images of segments $[0,1]$ and $[0, \tau]$ in $X$, respectively.

2.2. Extended mapping class group. The extended mapping class group $\operatorname{Mod}(S)$ is the group of isotopy classes of (not necessarily orientation preserving) homeomorphisms of $S$ onto itself. The outer automorphism of $\pi_{1}(S)$ is $\operatorname{Out}\left(\pi_{1}(S)\right)=\operatorname{Aut}\left(\pi_{1}(S)\right) / \operatorname{Inn}\left(\pi_{1}(S)\right)$, where $\operatorname{Aut}\left(\pi_{1}(S)\right)$ and $\operatorname{Inn}\left(\pi_{1}(S)\right)$ denote the automorphism group and the inner automorphism group of $\pi_{1}(S)$, respectively.

We can identify $\operatorname{Mod}(S)$ with $\operatorname{Out}\left(\pi_{1}(S)\right)$. In fact, for an arbitrary homeomorphism $h$ of $S$ onto itself, and for any path $\delta$ connecting the base point $p \in S$ to $h(p)$, we have an automorphism $\left(h_{\delta}\right)_{*} \in \operatorname{Aut}\left(\pi_{1}(S)\right)$ which is defined by $\left(h_{\delta}\right)_{*}(\gamma)=\delta^{-1}(h \circ \gamma) \delta$. When we choose another path $\delta^{\prime}$ which connects $p$ to $h(p)$, then $\left(h_{\delta}\right)_{*}$ is conjugate to $\left(h_{\delta^{\prime}}\right)_{*}$ by $\delta^{-1} \delta^{\prime}$. The outer automorphism $h_{*} \in \operatorname{Out}\left(\pi_{1}(S)\right)$ is thus well defined independently of the choice of the path $\delta$. Furthermore $h$ is homotopic to the identity if and only if it acts trivially on $\pi_{1}(S)$. Therefore the map $\operatorname{Mod}(S) \rightarrow \operatorname{Out}\left(\pi_{1}(S)\right)$ defined as above is a homomorphism (see chapter 8 in [3] for more details). 
By fixing a basis of $H_{1}(S, \mathbb{Z}) \approx \mathbb{Z}^{2}$, we can also identify $\operatorname{Mod}(S)$ with $G L(2, \mathbb{Z})$ (see for example [3] P.231). In fact, when we fix a pair $(\alpha, \beta)$ of generators of $H_{1}(S, \mathbb{Z})$, we identify $h \in \operatorname{Mod}(S)$ with $\left(\begin{array}{ll}a & b \\ c & d\end{array}\right) \in G L(2, \mathbb{Z})$ if $h$ induces an isomorphism $h_{*}: H_{1}(S, \mathbb{Z}) \rightarrow$ $H_{1}(S, \mathbb{Z})$ such that $h_{*}(\alpha)=a \alpha+c \beta$ and $h_{*}(\beta)=b \alpha+d \beta$. Then we often identify the following three groups:

$$
\operatorname{Out}\left(\pi_{1}(S)\right) \cong \operatorname{Mod}(S) \cong G L(2, \mathbb{Z}) .
$$

Let $\mathrm{Mod}^{+}(S)\left(\right.$ resp. $\left.\operatorname{Mod}^{-}(S)\right)$ denote the subset of $\operatorname{Mod}(S)$ consisting of isotopy classes of orientation preserving (resp. reversing) homeomorphisms from $S$ to itself. In particular, we can identify $\operatorname{Mod}^{+}(S)$ with $S L(2, \mathbb{Z})$. An element $\varphi \in \operatorname{Mod}^{+}(S)$ induces a map $\mathcal{T}(S) \rightarrow$ $\mathcal{T}(S)$ given by sending $X=[X, f]$ to $\varphi \cdot X:=\left[X, f \circ \varphi_{*}^{-1}\right]$, where $\varphi_{*} \in \operatorname{Out}\left(\pi_{1}(S)\right)$ denotes the element corresponding to $\varphi$ by the above identification. Let $\bar{S}$ be $S$ with its orientation reversed. Then $\varphi \in \operatorname{Mod}^{-}(S)$ induces a map $\mathcal{T}(S) \rightarrow \mathcal{T}(\bar{S})$ in a similar way.

2.3. Action of $\operatorname{Mod}^{+}(S)$ on $\mathcal{T}(S)$. The action of $\operatorname{Mod}^{+}(S)$ on $\mathcal{T}(S)$ can be identified with the action of $S L(2, \mathbb{Z})$ on $\mathbb{H}$ by Möbius transformation (see [3], P.346):

$$
\left(\begin{array}{ll}
a & b \\
c & d
\end{array}\right) \mapsto f(z)=\frac{a z-b}{-c z+d} .
$$

Note that $\varphi \in \operatorname{Mod}^{+}(S)$ acts on $\mathcal{T}(S)$ trivially if and only if it corresponds to $\pm I$ under the identification $\operatorname{Mod}^{+}(S) \cong S L(2, \mathbb{Z})$. A fundamental domain for the action of $S L(2, \mathbb{Z})$ on $\mathbb{H}$ is

$$
K=\left\{z \in \mathbb{H}:-\frac{1}{2} \leq \Re z \leq \frac{1}{2},|z| \geq 1\right\}
$$

In $K$, there are only three points $i, e^{i \pi / 3}$ and $e^{2 i \pi / 3}$ that are fixed by some element of $S L(2, \mathbb{Z})$.

2.4. Quasi-fuchsian space and Earle slices. The quasi-fuchsian space $\mathcal{Q F}(S)$ of $S$ is defined to be the set of conjugacy classes of faithful and discrete representations $\rho$ : $\pi_{1}(S) \rightarrow P S L(2, \mathbb{C})$ such that the image of the loop around the puncture is parabolic and that the image $\Gamma:=\rho\left(\pi_{1}(S)\right)$ is quasi-fuchsian. The region of discontinuity $\Omega$ of $\Gamma$ has exactly two simply connected invariant components $\Omega_{ \pm}$. We choose the labeling \pm in such a way that the homotopy basis of $\Omega_{+} / \Gamma$ induced by the ordered pair $(\rho(\alpha), \rho(\beta))$ is canonical with respect to the orientation of $\Omega_{+}$.

Earle slices for once punctured torus are defined by the following theorem.

THEOREM 2.1 ([2], [6]). Let $S$ be a once punctured torus, and $\mathcal{T}(S)$ the Teichmüller space of $S$. Then for any $X \in \mathcal{T}(S)$ and an automorphism $\theta$ of $\pi_{1}(S)$ induced by an orientation reversing diffeomorphism of $S$, there exists a unique representation $\rho: \pi_{1}(S) \rightarrow \Gamma$ in the quasi-fuchsian space $\mathcal{Q F}(S)$, such that

1. $\Omega_{+} / \Gamma=X$ in $\mathcal{T}(S)$, where the marking of $\Omega_{+} / \Gamma$ is induced from $\rho$, and 
2. there exists a conformal map $F: \Omega_{+} \rightarrow \Omega_{-}$such that $F \circ \rho(\gamma)=\rho(\theta(\gamma)) \circ F$ for all $\gamma \in \pi_{1}(S)$.

Furthermore, if $\theta^{2}=i d$, then $F$ is a Möbius transformation of order two.

We denote the representation $\rho$ obtained in Theorem 2.1 by $\rho_{\theta, X}$. For a given automorphism $\theta$ of $\pi_{1}(S)$ induced by an orientation reversing diffeomorphism of $S$, the Earle slice $\mathcal{E}_{\theta}$ of $\mathcal{Q F}(S)$ is defined to be the subset

$$
\mathcal{E}_{\theta}:=\left\{\rho_{\theta, X} \mid X \in \mathcal{T}(S)\right\}
$$

of $\mathcal{Q F}(S)$.

\section{Classification of Earle slices associated with involutions}

Let $\rho \in \mathcal{Q F}(S)$ and $\Gamma=\rho\left(\pi_{1}(S)\right)$. The Kleinian manifold $\bar{M}=\left(\mathbb{H}^{3} \cup \Omega\right) / \Gamma$ is bounded by a pair of Riemann surfaces denoted by $X$ and $Y$. The orientations of $X$ and $Y$ are induced from that of $\Omega$. There is a homeomorphism $\psi: S \times[0,1] \rightarrow \bar{M}$ such that $\psi_{*}=\rho$. After equipping $X$ and $Y$ with markings $\left.\psi\right|_{S \times\{0\}}: S \rightarrow X$ and $\left.\psi\right|_{S \times\{1\}}: \bar{S} \rightarrow Y$, respectively, we can regard $X \in \mathcal{T}(S)$ and $Y \in \mathcal{T}(\bar{S})$. The map $\mathcal{Q F}(S) \rightarrow \mathcal{T}(S) \times \mathcal{T}(\bar{S})$, $\rho \mapsto(X, Y)$ defined as above has been proved to be a homeomorphism by Bers. So we denote by $Q(X, Y):=\rho \in \mathcal{Q F}(S)$ the preimage of $(X, Y)$.

Next we use this fact to interpret the definition of Earle slices.

THEOREM 3.1. For every automorphism $\theta$ of $\pi_{1}(S)$ induced by an orientation reversing diffeomorphism of $S$, the Earle slice $\mathcal{E}_{\theta}$ can be written as

$$
\mathcal{E}_{\theta}=\{Q(X, \theta \cdot X) \mid X \in \mathcal{T}(S)\} .
$$

Recall that $\theta$ induces a map $\mathcal{T}(S) \rightarrow \mathcal{T}(\bar{S})$ given by sending $X=[X, f]$ to $\theta \cdot X=$ $\left[X, f \circ \theta^{-1}\right]$.

Proof. Let $\rho \in \mathcal{E}_{\theta}$. By Theorem 2.1, there exists a conformal map $F: \Omega_{+} \rightarrow \Omega_{-}$, such that $F \circ \rho(\gamma)=\rho(\theta(\gamma)) \circ F$ for all $\gamma \in \pi_{1}(S)$. The conformal map $F$ induces an orientation preserving conformal map $\underline{\mathrm{F}}: \Omega_{+} / \Gamma \rightarrow \Omega_{-} / \Gamma$ such that $\underline{\mathrm{F}}_{*}(\rho(\gamma))=\rho(\theta(\gamma))$. We equip $\Omega_{+} / \Gamma$ and $\Omega_{-} / \Gamma$ with markings induced by $\rho$. We regard the marking $\rho: \pi_{1}(S) \rightarrow$ $\Gamma=\pi_{1}\left(\Omega_{+} / \Gamma\right)$ of $\Omega_{+} / \Gamma$ as induced from an orientation preserving homeomorphism $S \rightarrow$ $\Omega_{+} / \Gamma$. On the other hand, we regard the marking $\rho: \pi_{1}(S)=\pi_{1}(\bar{S}) \rightarrow \Gamma=\pi_{1}\left(\Omega_{-} / \Gamma\right)$ of $\Omega_{-} / \Gamma$ as induced from an orientation preserving homeomorphism $\bar{S} \rightarrow \Omega_{-} / \Gamma$. Thus one can see that $X=\left(\Omega_{+} / \Gamma, \rho\right) \in \mathcal{T}(S)$ and $Y=\left(\Omega_{-} / \Gamma, \rho\right) \in \mathcal{T}(\bar{S})$.

From the definition of Teichmüller space, we have $X=\left[\Omega_{+} / \Gamma, \rho\right]=\left[\Omega_{-} / \Gamma, \underline{\mathrm{F}}_{*} \circ \rho\right]=$ $\left[\Omega_{-} / \Gamma, \rho \circ \theta\right]$. So

$$
\theta \cdot X=\theta \cdot\left[\Omega_{-} / \Gamma, \rho \circ \theta\right]=\left[\Omega_{-} / \Gamma, \rho \circ \theta \circ \theta^{-1}\right]=\left[\Omega_{-} / \Gamma, \rho\right]=Y .
$$

Thus we obtain $\rho=Q(X, Y)=Q(X, \theta \cdot X)$. 
Let

$$
\operatorname{Inv}^{-}(S):=\left\{\varphi \in \operatorname{Mod}^{-}(S) \mid \varphi^{2}=i d\right\}
$$

and

$$
\operatorname{Inv}^{-}(S)_{*}:=\left\{\varphi_{*} \in \operatorname{Out}\left(\pi_{1}(S)\right) \mid \varphi \in \operatorname{Inv}^{-}(S)\right\} .
$$

Under the identification $\operatorname{Out}\left(\pi_{1}(S)\right) \cong G L(2, \mathbb{Z})$, we have the following bijective correspondence:

$$
\operatorname{Inv}^{-}(S)_{*} \longleftrightarrow\left\{\left(\begin{array}{cc}
a & b \\
c & -a
\end{array}\right) \in G L(2, \mathbb{Z}): a^{2}+b c=1\right\}
$$

In this paper, we focus on Earle slices $\mathcal{E}_{\theta}$ associated with $\theta \in \operatorname{Inv}^{-}(S)_{*}$.

We have the following classification of elements of $\operatorname{Inv}^{-}(S)_{*}$, which is deduced from Theorem 3.4 below.

THEOREM 3.2. Let $\theta \in \operatorname{Inv}^{-}(S)_{*}$, then exactly one of the following is satisfied:

1. There exists a pair $(\alpha, \beta)$ of canonical generators of $\pi_{1}(S)$ such that $\theta(\alpha)=\alpha$, $\theta(\beta)=\beta^{-1}$.

2. There exists a pair $(\alpha, \beta)$ of canonical generators of $\pi_{1}(S)$ such that $\theta(\alpha)=\beta$, $\theta(\beta)=\alpha$.

DEFINITION 3.3 ([5], [6], [7]). We say that $\theta$ is rectangular, if $\theta$ satisfies condition 1 of Theorem 3.2. The corresponding Earle slice $\mathcal{E}_{\theta}$ is called rectangular Earle slice. We say $\theta$ is rhombic if $\theta$ satisfies condition 2 of Theorem 3.2. The corresponding Earle slice $\mathcal{E}_{\theta}$ is called rhombic Earle slice.

An ordered pair $\left(\omega_{1}, \omega_{2}\right)$ of $\mathbb{Z}^{2}$ is canonical if $\operatorname{det}\left(\omega_{1}, \omega_{2}\right)=1$. We obtain Theorem 3.2 from the bijective correspondence $\operatorname{Out}\left(\pi_{1}(S)\right) \cong G L(2, \mathbb{Z})$ and the following theorem.

Theorem 3.4 ([4] P.166, Lemma 5.5). For $A \in G L(2, \mathbb{Z})$ with $\operatorname{det}(A)=-1$ and $A^{2}=E$, exactly one of the following is satisfied:

1. There exists a pair $\left(\omega_{1}, \omega_{2}\right)$ of canonical generators of $\mathbb{Z}^{2}$ such that $A \omega_{1}=\omega_{1}$ and $A \omega_{2}=-\omega_{2}$.

2. There exists a pair $\left(\omega_{1}, \omega_{2}\right)$ of canonical generators of $\mathbb{Z}^{2}$ such that $A \omega_{1}=\omega_{2}$ and $A \omega_{2}=\omega_{1}$.

REMARK. In Theorem 3.4, $A$ is conjugate to $\left(\begin{array}{cc}1 & 0 \\ 0 & -1\end{array}\right)$ in Case 1 , and is conjugate to $\left(\begin{array}{ll}0 & 1 \\ 1 & 0\end{array}\right)$ in Case 2

Throughout this paper, we denote $\theta_{1}=\left(\begin{array}{cc}1 & 0 \\ 0 & -1\end{array}\right)$ and $\theta_{2}=\left(\begin{array}{ll}0 & 1 \\ 1 & 0\end{array}\right)$. 
Lemma 3.5. Let $A=\left(\begin{array}{ll}a & b \\ c & d\end{array}\right) \in G L(2, \mathbb{Z})$ be as in Theorem 3.4. Then $A$ is conjugate to $\theta_{1}$ by some element of $S L(2, \mathbb{Z})$ if and only if both $b$ and $c$ are even.

Proof. Let $B=\left(\begin{array}{ll}p & q \\ r & s\end{array}\right) \in S L(2, \mathbb{Z})$. Since we have

$$
\begin{gathered}
B \theta_{1} B^{-1}=\left(\begin{array}{cc}
* & -2 p q \\
-2 r s & *
\end{array}\right), \\
B \theta_{2} B^{-1}=\left(\begin{array}{cc}
* & p^{2}-q^{2} \\
s^{2}-r^{2} & *
\end{array}\right),
\end{gathered}
$$

the "only if" part is trivial.

We only need to show that either $p^{2}-q^{2}$ or $s^{2}-r^{2}$ is odd. Suppose for contradiction that both $p^{2}-q^{2}$ and $s^{2}-r^{2}$ are even. Then $p$ and $q$ have the same parity, and so do $s$ and $r$. Then $p s$ and $q r$ have the same parity. This contradicts $p s-q r=1$.

\section{Stabilizers of Earle slices}

A mapping class $\varphi \in \operatorname{Mod}^{+}(S)$ acts on $\mathcal{Q F}(S)$ by sending a representation $Q(X, Y)$ to $\varphi \cdot Q(X, Y):=Q(X, Y) \circ \varphi_{*}^{-1}$. This action is compatible with the actions of $\operatorname{Mod}^{+}(S)$ on $\mathcal{T}(S)$ and $\mathcal{T}(\bar{S})$; that is

$$
\varphi \cdot Q(X, Y)=Q(\varphi \cdot X, \varphi \cdot Y) .
$$

In this chapter, we will study the action of $\operatorname{Mod}^{+}(S)$ on the set of Earle slices of $\mathcal{Q F}(S)$. Especially, we will give the stabilizer subgroup of each Earle slice in $\operatorname{Mod}^{+}(S) \cong S L(2, \mathbb{Z})$.

LeMma 4.1. Let $\theta, \theta^{\prime} \in \operatorname{Inv}^{-}(S)_{*}$. Then $\mathcal{E}_{\theta}=\mathcal{E}_{\theta^{\prime}}$ if and only if $\theta= \pm \theta^{\prime}$ in $G L(2, \mathbb{Z})$.

Proof. Suppose that $\mathcal{E}_{\theta}=\mathcal{E}_{\theta^{\prime}}$. Then $Q(X, \theta \cdot X)=Q\left(X, \theta^{\prime} \cdot X\right)$ for all $X \in \mathcal{T}(S)$. Then we have $\theta \cdot X=\theta^{\prime} \cdot X$ and hence $X=\theta^{-1} \cdot \theta^{\prime} \cdot X$ for all $X \in \mathcal{T}(S)$. From subsection 2.3, we know that an element in $\operatorname{Mod}^{+}(S)$ acts on $\mathcal{T}(S)$ trivially if and only if it corresponds to $\pm I$. Thus $\theta^{-1} \circ \theta^{\prime}= \pm I$ in $S L(2, \mathbb{Z})$. That means $\theta= \pm \theta^{\prime}$ in $G L(2, \mathbb{Z})$.

The converse is trivial.

THEOREM 4.2. Let $\theta \in \operatorname{Inv}^{-}(S)_{*}$ and $r \in \operatorname{Mod}^{+}(S)$. Then we have the following:

(a) The $\operatorname{set} r\left(\mathcal{E}_{\theta}\right)=\left\{\rho \circ r_{*}^{-1} \mid \rho \in \mathcal{E}_{\theta}\right\}$ is equal to $\mathcal{E}_{\theta^{\prime}}$ where $\theta^{\prime}=r_{*} \circ \theta \circ r_{*}^{-1}$.

(b) $\mathcal{E}_{\theta}$ is rhombic (resp. rectangular) if and only if $r\left(\mathcal{E}_{\theta}\right)$ is rhombic (resp. rectangular).

Proof. (a) By Theorem 3.1, we have

$$
r\left(\mathcal{E}_{\theta}\right)=\{Q(r \cdot X, r \cdot \theta \cdot X) \mid X \in \mathcal{T}(S)\} .
$$


Since $r$ induces an automorphism of $\mathcal{T}(S)$, we obtain

$$
\{Q(r \cdot X, r \cdot \theta \cdot X) \mid X \in \mathcal{T}(S)\}=\left\{Q\left(X, r \cdot \theta \cdot r^{-1} \cdot X\right) \mid X \in \mathcal{T}(S)\right\} .
$$

For all $X=[X, f] \in \mathcal{T}(S)$, we have

$$
\begin{aligned}
r \cdot \theta \cdot r^{-1} \cdot X=\left[X, f \circ r_{*} \circ \theta^{-1} \circ r_{*}^{-1}\right] & =\left[X, f \circ\left(r_{*} \circ \theta \circ r_{*}^{-1}\right)^{-1}\right] \\
& =\theta^{\prime} \cdot X,
\end{aligned}
$$

thus $r\left(\mathcal{E}_{\theta}\right)=\mathcal{E}_{\theta^{\prime}}$.

(b) It follows from (a) and the definitions of the two kinds of Earle slices.

By Theorem 3.4, $\theta \in \operatorname{Inv}^{-}(S)_{*}$ is conjugate to $\theta_{1}$ or $\theta_{2}$ in $G L(2, \mathbb{Z})$ by some element in $S L(2, \mathbb{Z})$. Therefore we have the following corollary.

COROLlary 4.3. For any $\theta \in \operatorname{Inv}^{-}(S)_{*}$, there exists $r \in \operatorname{Mod}^{+}(S)$ such that $\mathcal{E}_{\theta}=$ $r\left(\mathcal{E}_{\theta_{1}}\right)$ or $\mathcal{E}_{\theta}=r\left(\mathcal{E}_{\theta_{2}}\right)$.

THEOREM 4.4. For every Earle slice $\mathcal{E}_{\theta}$, we have

$$
\operatorname{Stab}_{\operatorname{Mod}^{+}(S)} \mathcal{E}_{\theta}=\left\{ \pm I, \pm g\left(\begin{array}{cc}
0 & 1 \\
-1 & 0
\end{array}\right) g^{-1}\right\},
$$

where $g$ is an element of $S L(2, \mathbb{Z})$ such that $\theta=g \theta_{1} g^{-1}$ if $\theta$ is rectangular, or $\theta=g \theta_{2} g^{-1}$ if $\theta$ is rhombic.

PROOF. If $\mathcal{E}_{\theta}=r\left(\mathcal{E}_{\theta}\right)$, it follows from Theorem 4.2 that $r\left(\mathcal{E}_{\theta}\right)=\mathcal{E}_{r_{*} \circ \theta \circ r_{*}^{-1}}$. Then by Lemma 4.1, we have $\theta= \pm r_{*} \circ \theta \circ r_{*}^{-1}$ in $G L(2, \mathbb{Z})$.

We first assume that $\theta=\theta_{1}$. Let $r_{*}=\left(\begin{array}{ll}a & b \\ c & d\end{array}\right) \in S L(2, \mathbb{Z})$. By a calculation, we obtain from $\theta= \pm r_{*} \circ \theta \circ r_{*}^{-1}$ that $r_{*}= \pm I, \pm\left(\begin{array}{cc}0 & 1 \\ -1 & 0\end{array}\right)$. Similarly, when $\theta=\theta_{2}$, we obtain the same result $r_{*}= \pm I, \pm\left(\begin{array}{cc}0 & 1 \\ -1 & 0\end{array}\right)$.

In general, by Theorem $3.4, \theta$ is conjugate to $\theta_{1}$ or $\theta_{2}$ by some element of $S L(2, \mathbb{Z})$. Suppose $\theta=g \theta_{i} g^{-1}$ for some $g \in S L(2, \mathbb{Z})$, where $i=1$ or 2 . Combining with $r_{*} \theta r_{*}^{-1}=$ $\pm \theta$, we obtain

$$
\left(g^{-1} r_{*} g\right) \theta_{i}\left(g^{-1} r_{*} g\right)^{-1}= \pm \theta_{i}
$$

It follows from the discussion above that $g^{-1} r_{*} g= \pm I, \pm\left(\begin{array}{cc}0 & 1 \\ -1 & 0\end{array}\right)$. Thus we obtain

$$
r_{*}= \pm I, \pm g\left(\begin{array}{cc}
0 & 1 \\
-1 & 0
\end{array}\right) g^{-1}
$$




\section{Intersection of two Earle slices}

Recall that the Teichmüller space $\mathcal{T}(S)$ of once punctured torus $S$ can be identified with the upper half space $\mathbb{H}$ after fixing a pair of generators of $\pi_{1}(S)$. Let $S_{z}$ denote the element in $\mathcal{T}(S)$ corresponding to $z \in \mathbb{H}$. Let

$$
C=\left(\begin{array}{cc}
0 & 1 \\
-1 & 0
\end{array}\right), D=\left(\begin{array}{cc}
1 & -1 \\
1 & 0
\end{array}\right) \in S L(2, \mathbb{Z}) .
$$

Note that $C^{2}=D^{3}=I$ in $S L(2, \mathbb{Z}) /\{ \pm I\}$. Since $S L(2, \mathbb{Z})$ act on $\mathbb{H}$, we have $C(i)=i$, $D^{ \pm 1}\left(e^{i \pi / 3}\right)=e^{i \pi / 3}$. So if we regard $C$ and $D^{ \pm 1}$ as elements of $\operatorname{Mod}^{+}(S)$ acting on $\mathcal{T}(S)$, we can say that $C$ fixes $S_{i} \in \mathcal{T}(S)$ and $D^{ \pm 1}$ fixes $S_{e^{i \pi / 3}} \in \mathcal{T}(S)$. Conversely $\varphi \in \operatorname{Mod}^{+} S \cong$ $S L(2, \mathbb{Z})$ has a fixed point in $\mathcal{T}(S)$, then $\varphi$ is conjugate to $\pm C$ or $\pm D$ or $\pm D^{-1}$. In what follows, we will always let $C$ and $D$ be as above.

We first give a necessary and sufficient condition for $\mathcal{E}_{\theta} \cap \mathcal{E}_{\theta^{\prime}} \neq \emptyset$.

THEOREM 5.1. Let $\theta, \theta^{\prime} \in \operatorname{Inv}^{-}(S)_{*}$ with $\theta \neq \pm \theta^{\prime}$. Then $\mathcal{E}_{\theta} \cap \mathcal{E}_{\theta^{\prime}} \neq \emptyset$ if and only if $\theta^{-1} \theta^{\prime}$ is conjugate in $S L(2, \mathbb{Z})$ to $\pm C$ or $\pm D$ or $\pm D^{-1}$. Moreover, if $\theta^{-1} \theta^{\prime}= \pm g C g^{-1}$ for some $g \in S L(2, \mathbb{Z})$, then

$$
\mathcal{E}_{\theta} \cap \mathcal{E}_{\theta^{\prime}}=\left\{Q\left(S_{g(i)}, \theta \cdot S_{g(i)}\right)\right\}
$$

and if $\theta^{-1} \theta^{\prime}$ is equal to $\pm g D g^{-1}$ or $\pm g D^{-1} g^{-1}$, then

$$
\mathcal{E}_{\theta} \cap \mathcal{E}_{\theta^{\prime}}=\left\{Q\left(S_{g\left(e^{i \pi / 3}\right)}, \theta \cdot S_{g\left(e^{i \pi / 3}\right)}\right)\right\} .
$$

Proof. If $\mathcal{E}_{\theta} \cap \mathcal{E}_{\theta^{\prime}} \neq \emptyset$, then there exists $X=[X, f] \in \mathcal{T}(S)$ such that $\theta \cdot X=\theta^{\prime} \cdot X$ and hence that $X=\theta^{-1} \cdot \theta^{\prime} \cdot X$. So $\theta^{-1} \theta^{\prime}$ fixes the point $[X, f]$. By identifying $\operatorname{Mod}^{+}(S)$ with $S L(2, \mathbb{Z})$, we have that $\theta^{-1} \theta^{\prime}$ is conjugate to $\pm C$ or $\pm D$ or $\pm D^{-1}$ in $S L(2, \mathbb{Z})$.

When $\theta^{-1} \theta^{\prime}= \pm g C g^{-1}$ for some $g \in S L(2, \mathbb{Z}), \theta^{-1} \theta^{\prime}$ fixes $S_{g(i)}$. Therefore $\mathcal{E}_{\theta} \cap \mathcal{E}_{\theta^{\prime}}=$ $\left\{Q\left(S_{g(i)}, \theta \cdot S_{g(i)}\right)\right\}$. Similarly, if $\theta^{-1} \theta^{\prime}= \pm g D g^{-1}$ or $\pm g D^{-1} g^{-1}$, we have $\mathcal{E}_{\theta} \cap \mathcal{E}_{\theta^{\prime}}=$ $\left\{Q\left(S_{g\left(e^{i \pi / 3}\right)}, \theta \cdot S_{g\left(e^{i \pi / 3}\right)}\right)\right\}$.

ExAmPles. (1) Since $\theta_{2}^{-1} \theta_{1}=C$, we have $\mathcal{E}_{\theta_{1}} \cap \mathcal{E}_{\theta_{2}}=\left\{Q\left(S_{i}, \theta_{1} \cdot S_{i}\right)\right\}$.

(2) Since $\theta_{2} D$ is conjugate to $\theta_{2}$ by $\left(\begin{array}{ll}1 & 1 \\ 0 & 1\end{array}\right) \in S L(2, \mathbb{Z})$, we know that $\theta_{2} D$ is in $\operatorname{Inv}^{-1}(S)_{*}$ and is rhombic. We have $\mathcal{E}_{\theta_{2}} \cap \mathcal{E}_{\theta_{2} D}=\left\{Q\left(S_{e^{i \pi / 3}}, \theta_{2} \cdot S_{e^{i \pi / 3}}\right)\right\}$ since $\theta_{2}^{-1}\left(\theta_{2} D\right)=D$. Similarly, we have $\mathcal{E}_{\theta_{2}} \cap \mathcal{E}_{\theta_{2} D^{-1}}=\left\{Q\left(S_{e^{i \pi / 3}}, \theta_{2} \cdot S_{e^{i \pi / 3}}\right)\right\}$. Therefore,

$$
\mathcal{E}_{\theta_{2}} \cap \mathcal{E}_{\theta_{2} D} \cap \mathcal{E}_{\theta_{2} D^{-1}}=\left\{Q\left(S_{e^{i \pi / 3}}, \theta_{2} \cdot S_{e^{i \pi / 3}}\right)\right\} .
$$

THEOREM 5.2. Let $\theta, \theta^{\prime} \in \operatorname{Inv}^{-}(S)_{*}$ with $\theta \neq \pm \theta^{\prime}$ and assume that $\mathcal{E}_{\theta} \cap \mathcal{E}_{\theta^{\prime}} \neq \emptyset$. Then $\theta^{-1} \theta^{\prime}$ is conjugate to $\pm D$ or $\pm D^{-1}$ in $S L(2, \mathbb{Z})$ if and only if both $\theta$ and $\theta^{\prime}$ are rhombic. 
Proof. First, we assume that $\theta^{-1} \theta^{\prime}$ is conjugate to $\pm D$ in $S L(2, \mathbb{Z})$. The proof for $\pm D^{-1}$ is similar. Since $\theta \in \operatorname{Inv}^{-}(S)_{*}$, we can let $\theta=\left(\begin{array}{cc}a & b \\ c & -a\end{array}\right) \in G L(2, \mathbb{Z})$ with $a^{2}+b c=$ 1.

Suppose first that $\theta^{-1} \theta^{\prime}= \pm D$. Then we have $\theta^{\prime}= \pm \theta D= \pm\left(\begin{array}{cc}a+b & -a \\ c-a & -c\end{array}\right)$. Since $\theta^{\prime} \in \operatorname{Inv}^{-}(S)_{*}$, we obtain $a+b-c=0$. Suppose for contradiction that $\theta$ is rectangular. It follows immediately from Lemma 3.5, both $b$ and $c$ are even. By $a+b-c=0, a$ is also even. Hence $a^{2}+b c \neq 1$, which is a contradiction. Thus $\theta$ is rhombic. The proof for $\theta^{\prime}$ is parallel.

In general, $\theta^{\prime}= \pm \theta g D g^{-1}$ for some $g \in S L(2, \mathbb{Z})$. Then we have $g^{-1} \theta^{\prime} g= \pm g^{-1} \theta g D$. One can see that $g^{-1} \theta g$ and $g^{-1} \theta^{\prime} g$ are rhombic from the discussion for $\theta^{-1} \theta^{\prime}= \pm D$. Therefore, both $\theta$ and $\theta^{\prime}$ are rhombic.

Next we will show the necessity. By Theorem 5.1, $\mathcal{E}_{\theta} \cap \mathcal{E}_{\theta^{\prime}} \neq \emptyset$ implies that $\theta^{-1} \theta^{\prime}$ is conjugate to $\pm C$ or $\pm D$ or $\pm D^{-1}$. Since $\theta$ and $\theta^{\prime}$ are rhombic, by taking a conjugation if necessary, we may assume $\theta=\theta_{2}$ and $\theta^{\prime}=\left(\begin{array}{cc}a & b \\ c & -a\end{array}\right)$ with $a^{2}+b c=1$. Then we have $\theta_{2}^{-1} \theta^{\prime}=\left(\begin{array}{cc}c & -a \\ a & b\end{array}\right)$. Suppose for contradiction that $\theta_{2}^{-1} \theta^{\prime}$ is conjugate to $\pm C$. Then $\operatorname{Tr} \theta_{2}^{-1} \theta^{\prime}=b+c=0$. Then from $a^{2}+b c=1$ we have $a^{2}-b^{2}=1$. Therefore $a= \pm 1$ and $b=c=0$. So $\theta^{\prime}= \pm \theta_{1}$. It contradicts the assumption that $\theta^{\prime}$ is rhombic. Thus $\theta^{-1} \theta^{\prime}$ is conjugate to $\pm D$ or $\pm D^{-1}$.

Next we will study the case that both $\theta$ and $\theta^{\prime}$ are rectangular.

THEOREM 5.3. If $\theta, \theta^{\prime} \in \operatorname{Inv}^{-}(S)_{*}$ are rectangular and $\theta \neq \pm \theta^{\prime}$, then $\mathcal{E}_{\theta} \cap \mathcal{E}_{\theta^{\prime}}=\emptyset$.

Proof. Suppose that $\mathcal{E}_{\theta} \cap \mathcal{E}_{\theta^{\prime}} \neq \emptyset, \theta \neq \pm \theta^{\prime}$, and $\theta$ is rectangular. We will show that $\theta^{\prime}$ is not rectangular.

By Theorem 5.1 and Theorem 5.2, we know that $\theta^{-1} \theta^{\prime}$ is conjugate to $\pm C$ in $S L(2, \mathbb{Z})$. Let $\theta^{-1} \theta^{\prime}=g C g^{-1}$, where $g=\left(\begin{array}{ll}a & b \\ c & d\end{array}\right) \in S L(2, \mathbb{Z})$. We first suppose that $\theta=\theta_{1}$. Then $\theta^{\prime}= \pm \theta_{1} g C g^{-1}= \pm\left(\begin{array}{cc}-b d-a c & b^{2}+a^{2} \\ d^{2}+c^{2} & -b d-a c\end{array}\right)$. By Lemma 3.5, one can see that $\theta^{\prime}$ is not rectangular. In fact, if both $b^{2}+a^{2}$ and $d^{2}+c^{2}$ are even, then $a$ and $b$ have the same parity, so do $d$ and $c$. Therefore $a d$ and $b c$ have the same parity. Thus $a d-b c$ is even. It contradicts the fact that $g \in S L(2, \mathbb{Z})$.

In general, $\theta=h \theta_{1} h^{-1}$ for some $h \in S L(2, \mathbb{Z})$. Then we have $\theta^{\prime}= \pm h \theta_{1} h^{-1} g C g^{-1}$, that is $h^{-1} \theta^{\prime} h= \pm \theta_{1}\left(h^{-1} g\right) C\left(h^{-1} g\right)^{-1}$. From the discussion above, $h^{-1} \theta^{\prime} h$ is not rectangular and hence $\theta^{\prime}$ is not rectangular. 
From Theorem 5.1, Theorem 5.2 and Theorem 5.3, we obtain the following corollary.

COROLlary 5.4. Let $\theta, \theta^{\prime} \in \operatorname{Inv}^{-}(S)_{*}$ with $\theta \neq \pm \theta^{\prime}$ and assume that $\mathcal{E}_{\theta} \cap \mathcal{E}_{\theta^{\prime}} \neq \emptyset$. Then $\theta^{-1} \theta^{\prime}$ is conjugate to $\pm C$ in $S L(2, \mathbb{Z})$ if and only if one of $\theta$ and $\theta^{\prime}$ is rectangular and the other is rhombic.

In the following, we will study for a given Earle slice, how many Earle slices intersect with it.

THEOREM 5.5. For any rhombic (resp. rectangular) Earle slice $\mathcal{E}_{\theta}$, there exists a unique rectangular (resp. rhombic) Earle slice $\mathcal{E}_{\theta^{\prime}}$, such that $\mathcal{E}_{\theta} \cap \mathcal{E}_{\theta^{\prime}} \neq \emptyset$.

PROOF. We only consider the case of rhombic Earle slice. The proof for rectangular Earle slice is similar.

First, we show the existence. From Example (1), we see that for the rhombic Earle slice $\mathcal{E}_{\theta_{2}}$, there exists the rectangular Earle slice $\mathcal{E}_{\theta_{1}}$ such that $\mathcal{E}_{\theta_{1}} \cap \mathcal{E}_{\theta_{2}} \neq \emptyset$.

In general, for any rhombic Earle slice $\mathcal{E}_{h \theta_{2} h^{-1}}$ with $h \in S L(2, \mathbb{Z})$, there exists a rectangular Earle slice $\mathcal{E}_{h \theta_{1} h^{-1}}$ such that $\mathcal{E}_{h \theta_{2} h^{-1}} \cap \mathcal{E}_{h \theta_{1} h^{-1}} \neq \emptyset$, since $\left(h \theta_{2}^{-1} h^{-1}\right)\left(h \theta_{1} h^{-1}\right)=$ $h C h^{-1}$.

Next, we will show the uniqueness. By Corollary 5.4, if one rhombic Earle slice $\mathcal{E}_{\theta}$ intersects with one rectangular Earle slice $\mathcal{E}_{\theta^{\prime}}$, we have $\theta^{-1} \theta^{\prime}= \pm g C g^{-1}$ for some $g \in$ $S L(2, \mathbb{Z})$.

Suppose first that $\theta=\theta_{2}$. Let $\theta^{\prime}=\left(\begin{array}{ll}a & b \\ c & d\end{array}\right)$. Then $a=-d$ and $a^{2}+b c=1$. We also have $b+c=\operatorname{Tr}\left(\theta_{2}^{-1} \theta^{\prime}\right)=\operatorname{Tr}\left( \pm g C g^{-1}\right)= \pm \operatorname{Tr} C=0$. Thus one can see that $a=-d= \pm 1$ and $b=c=0$. It follows that $\theta^{\prime}= \pm \theta_{1}$. Therefore $\mathcal{E}_{\theta_{1}}$ is the unique rectangular Earle slice which satisfies the condition.

In general, $\theta=h \theta_{2} h^{-1}$ for some $h \in S L(2, \mathbb{Z})$. Then we have $\theta^{-1} \theta^{\prime}=h \theta_{2} h^{-1} \theta^{\prime}=$ $g C g^{-1}$ and so $\theta_{2}\left(h^{-1} \theta^{\prime} h\right)= \pm\left(h^{-1} g\right) C\left(h^{-1} g\right)^{-1}$. It follows from the discussion above that $h^{-1} \theta^{\prime} h=\theta_{1}$, that is $\theta^{\prime}=h \theta_{1} h^{-1}$. Thus for any rhombic Earle slice $\mathcal{E}_{h \theta_{2} h^{-1}}$ with $h \in$ $S L(2, \mathbb{Z})$, there exists a unique rectangular Earle slice $\mathcal{E}_{h \theta_{1} h^{-1}}$ such that $\mathcal{E}_{h \theta_{2} h^{-1}} \cap \mathcal{E}_{h \theta_{1} h^{-1}} \neq$ $\emptyset$.

THEOREM 5.6. For any rhombic Earle slice $\mathcal{E}_{\theta}$, there exist exactly four distinct rhombic Earle slices $\mathcal{E}_{\varphi_{i}}, i=1,2,3,4$ such that $\mathcal{E}_{\varphi_{i}} \cap \mathcal{E}_{\theta} \neq \emptyset$. Furthermore, the four Earle slices form two pairs, $\left\{\mathcal{E}_{\varphi_{1}}, \mathcal{E}_{\varphi_{2}}\right\}$ and $\left\{\mathcal{E}_{\varphi_{3}}, \mathcal{E}_{\varphi_{4}}\right\}$ such that:

$$
\begin{gathered}
\mathcal{E}_{\theta} \cap \mathcal{E}_{\varphi_{1}}=\mathcal{E}_{\theta} \cap \mathcal{E}_{\varphi_{2}} ; \\
\mathcal{E}_{\theta} \cap \mathcal{E}_{\varphi_{3}}=\mathcal{E}_{\theta} \cap \mathcal{E}_{\varphi_{4}} ; \\
\mathcal{E}_{\theta} \cap \mathcal{E}_{\varphi_{1}} \cap \mathcal{E}_{\varphi_{2}} \cap \mathcal{E}_{\varphi_{3}} \cap \mathcal{E}_{\varphi_{4}}=\emptyset .
\end{gathered}
$$


Proof. By Theorem 5.2, if two rhombic Earle slices $\mathcal{E}_{\theta}$ and $\mathcal{E}_{\theta^{\prime}}$ intersect, then $\theta^{-1} \theta^{\prime}$ is conjugate to $\pm D$ or $\pm D^{-1}$ in $S L(2, \mathbb{Z})$.

Suppose first that $\theta=\theta_{2}$. Let $\mathcal{E}_{\theta^{\prime}}$ be rhombic Earle slice such that $\mathcal{E}_{\theta} \cap \mathcal{E}_{\theta^{\prime}} \neq \emptyset$. One may assume that $\theta^{\prime}=\left(\begin{array}{cc}a & b \\ c & -a\end{array}\right)$ with $a^{2}+b c=1$. Since $\theta_{2}^{-1} \theta^{\prime}=\left(\begin{array}{cc}c & -a \\ a & b\end{array}\right)$ is conjugate to $\pm D$ or $\pm D^{-1}$, we have $\operatorname{Tr}\left(\theta_{2}^{-1} \theta^{\prime}\right)=b+c=1$ or -1 . By substituting this to the equation $a^{2}+b c=1$, we obtain $a^{2}-b^{2}+b=1$ or $a^{2}-b^{2}-b=1$.

Next, we will show how to find all integer solutions to $a^{2}-b^{2}+b=1$. It is equivalent to find all integer solutions to the equation $x^{2}-y^{2}+y=1$. Since the distance between two different integer numbers cannot be less than 1, one can see that the part of the curve $x^{2}-y^{2}+y=1$, which lies between the straight lines $y=x$ and $y=x+1$, has no integer solution(see Figure 1). So does for the part which lies between the straight lines $y=-x$ and $y=-x+1$. Then one can see from Figure 1 that all integer solutions to $x^{2}-y^{2}+y=1$ are $(x, y)=(1,0),(-1,0),(1,1)$ and $(-1,1)$. Therefore, all integer solutions for $a^{2}-b^{2}+b=1$ are $a= \pm 1, b=0$ and $a= \pm 1, b=1$. Since $b+c=1$ in this case, we obtain that $c=1$ when $b=0$, and $c=0$ when $b=1$.

Similarly, we know that all integer solutions to $a^{2}-b^{2}-b=1$ are $a= \pm 1, b=0$ and $a= \pm 1, b=-1$. By $b+c=-1$, one can see that $c=-1$ when $b=0$, and $c=0$ when $b=-1$.

From the above arguments, we obtain eight solutions $\pm \psi_{i}(i=1,2,3,4)$ for $\theta^{\prime}$, where

$$
\psi_{1}:=\left(\begin{array}{cc}
1 & 0 \\
1 & -1
\end{array}\right), \quad \psi_{2}:=\left(\begin{array}{cc}
-1 & 1 \\
0 & 1
\end{array}\right), \quad \psi_{3}:=\left(\begin{array}{cc}
1 & 1 \\
0 & -1
\end{array}\right), \quad \psi_{4}:=\left(\begin{array}{cc}
-1 & 0 \\
1 & 1
\end{array}\right) .
$$

Then $\theta^{\prime}$ can only be one of $\pm \psi_{i}$, where $i=1,2,3,4$. Since $\mathcal{E}_{\phi}=\mathcal{E}_{-\phi}$ for any $\phi \in \operatorname{Inv}^{-}(S)_{*}$, there exist exactly four distinct rhombic Earle slices intersect with $\mathcal{E}_{\theta_{2}}$.

Furthermore, we have

$$
\theta_{2}^{-1} \psi_{1}=D, \quad \theta_{2}^{-1} \psi_{2}=D^{-1}, \quad \theta_{2}^{-1} \psi_{3}=C D C^{-1}, \quad \theta_{2}^{-1} \psi_{4}=C D^{-1} C^{-1} .
$$

Therefore, one sees from Theorem 5.1 that they form two pairs, $\left\{\mathcal{E}_{\psi_{1}}, \mathcal{E}_{\psi_{2}}\right\}$ and $\left\{\mathcal{E}_{\psi_{3}}, \mathcal{E}_{\psi_{4}}\right\}$ which satisfy the desired properties.

In general, we suppose that $\theta=h \theta_{2} h^{-1}$ with $h \in S L(2, \mathbb{Z})$. Let $\mathcal{E}_{\theta^{\prime}}$ be rhombic Earle slice such that $\mathcal{E}_{\theta} \cap \mathcal{E}_{\theta^{\prime}} \neq \varnothing$. Since $\theta^{-1} \theta^{\prime}=h \theta_{2} h^{-1} \theta^{\prime}$ is conjugate to $\pm D$ or $\pm D^{-1}$, $\theta_{2}\left(h^{-1} \theta^{\prime} h\right)$ is also conjugate to $\pm D$ or $\pm D^{-1}$. It follows from the above discussion that $h^{-1} \theta^{\prime} h= \pm \psi_{i}, i=1,2,3$ or 4 . Thus $\theta^{\prime}= \pm h \psi_{i} h^{-1}, i=1,2,3$ or 4 . So for any rhombic Earle slice $\mathcal{E}_{h \theta_{2} h^{-1}}$, there exist exactly four rhombic Earle slices $\mathcal{E}_{h \psi_{i} h^{-1}}, i=1,2,3,4$ that satisfy the condition.

REMARK. In our new notation, Example (2) can be written as

$$
\mathcal{E}_{\theta_{2}} \cap \mathcal{E}_{\psi_{1}} \cap \mathcal{E}_{\psi_{2}}=\left\{Q\left(S_{e^{i \pi / 3}}, \theta_{2} \cdot S_{e^{i \pi / 3}}\right)\right\} .
$$




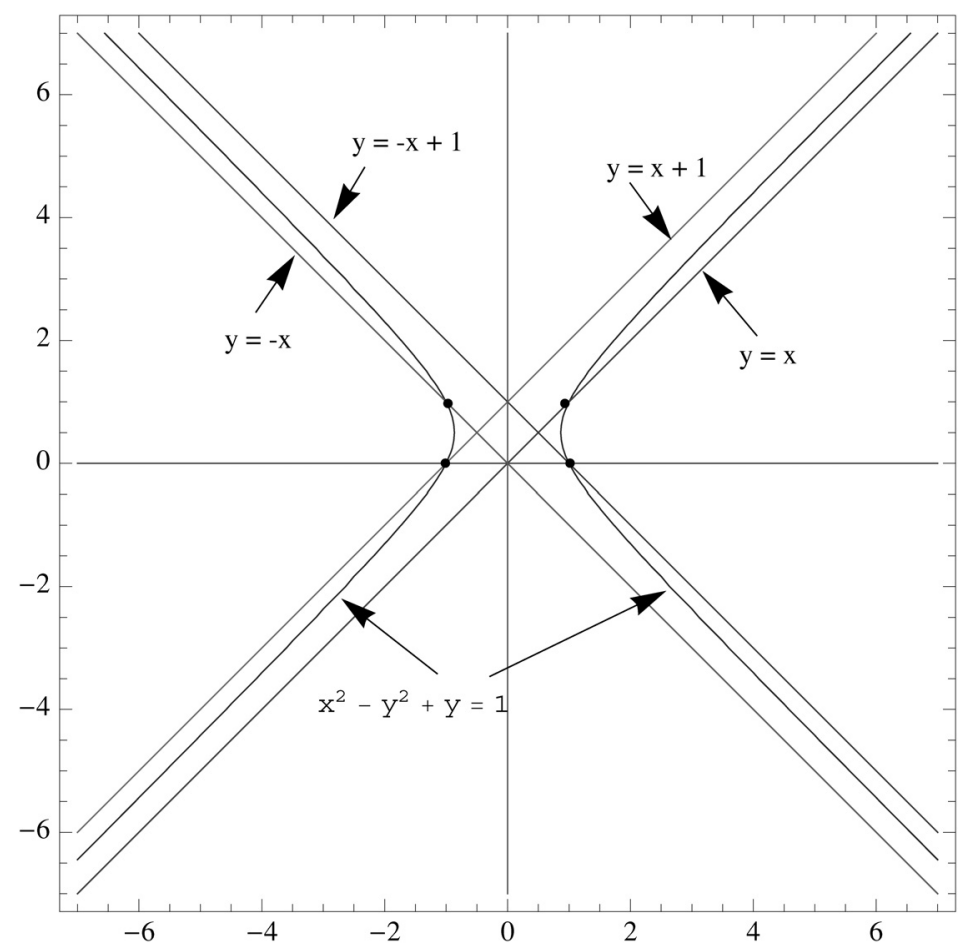

FIGURE 1. The curves defined by $x^{2}-y^{2}+y=1$ and the integer points.

Since $C\left(e^{i \pi / 3}\right)=e^{2 i \pi / 3}$, we have

$$
\mathcal{E}_{\theta_{2}} \cap \mathcal{E}_{\psi_{3}} \cap \mathcal{E}_{\psi_{4}}=\left\{Q\left(S_{e^{2 i \pi / 3}}, \theta_{2} \cdot S_{e^{2 i \pi / 3}}\right)\right\} .
$$

THEOREM 5.7. The union of all rhombic Earle slices is connected.

PROOF. It suffices to show that any rhombic Earle slice $\mathcal{E}_{g \theta_{2} g^{-1}}, g \in S L(2, \mathbb{Z})$, connects with $\mathcal{E}_{\theta_{2}}$ through a finite sequence of rhombic Earle slices.

From Theorem 5.6, we know that there exist exactly four distinct rhombic Earle slices $\mathcal{E}_{g \psi_{i} g^{-1}}, i=1,2,3,4$ such that $\mathcal{E}_{g \psi_{i} g^{-1}} \cap \mathcal{E}_{g \theta_{2} g^{-1}} \neq \emptyset$. Let $T:=\left(\begin{array}{ll}1 & 1 \\ 0 & 1\end{array}\right)$ and $S:=$ $\left(\begin{array}{ll}1 & 0 \\ 1 & 1\end{array}\right)$. By calculating, we obtain that

$$
\psi_{1}=T \theta_{2} T^{-1}, \quad \psi_{2}=S \theta_{2} S^{-1}, \quad \psi_{3}=S^{-1} \theta_{2} S, \quad \psi_{4}=T^{-1} \theta_{2} T .
$$

It is known that $S L(2, \mathbb{Z})$ is generated by $S$ and $T$. Therefore $g=s_{1} s_{2} \ldots s_{n}$ for some $n \in \mathbb{Z}^{+}$ 
with $s_{j} \in\left\{S, S^{-1}, T, T^{-1}\right\}$. Let $g_{k}=s_{1} s_{2} \ldots s_{k}$ for $1 \leqslant k \leqslant n$ and $g_{0}=I$. Then

$$
g_{k} \theta_{2} g_{k}^{-1}=g_{k-1} s_{k} \theta_{2} s_{k}^{-1} g_{k-1}^{-1}=g_{k-1} \psi_{i} g_{k-1}^{-1},
$$

where $i=1,2,3$ or 4 , which depends on $s_{k}$. It follows from the proof of Theorem 5.6 that

$$
\mathcal{E}_{g_{k} \theta_{2} g_{k}^{-1}} \cap \mathcal{E}_{g_{k-1} \theta_{2} g_{k-1}^{-1}}=\mathcal{E}_{g_{k-1} \psi_{i} g_{k-1}^{-1}} \cap \mathcal{E}_{g_{k-1} \theta_{2} g_{k-1}^{-1}}=g_{k-1}\left(\mathcal{E}_{\psi_{i}} \cap \mathcal{E}_{\theta_{2}}\right) \neq \emptyset
$$

Therefore $\mathcal{E}_{g \theta_{2} g^{-1}}$ connects with $\mathcal{E}_{\theta_{2}}$ through a finite sequence of rhombic Earle slices.

REMARK. From the relationship between $\psi_{i}$ and $\theta_{2}$, where $i=1,2,3,4$, we have

$$
\mathcal{E}_{\psi_{1}}=T\left(\mathcal{E}_{\theta_{2}}\right), \quad \mathcal{E}_{\psi_{2}}=S\left(\mathcal{E}_{\theta_{2}}\right), \quad \mathcal{E}_{\psi_{3}}=S^{-1}\left(\mathcal{E}_{\theta_{2}}\right), \quad \mathcal{E}_{\psi_{4}}=T^{-1}\left(\mathcal{E}_{\theta_{2}}\right) .
$$

Combining Theorem 5.4 and Theorem 5.7, we obtain the following.

COROLLARY 5.8. The union of all Earle slices is connected.

\section{Trace coordinates}

Let $\widetilde{\mathcal{Q F}}(S)$ be the set of conjugacy classes of type-preserving, faithful and irreducible representations $\rho$ of $\pi_{1}(S)$ to $S L(2, \mathbb{C})$ such that the images are discrete and quasi-fuchsian group. Then $\widetilde{\mathcal{Q F}}(S)$ is a natural covering of $\mathcal{Q F}(S)$. We denote the preimage of $\mathcal{E}_{\theta} \in \mathcal{Q F}(S)$ by in $\widetilde{\mathcal{Q F}}(S)$ by $\widetilde{\mathcal{E}_{\theta}}$. Fix a pair $(\alpha, \beta)$ of generators of $\pi_{1}(S)$ and let $\mu: \widetilde{\mathcal{Q F}}(S) \rightarrow \mathbb{C}^{3}$ be the map which sends $[\rho]$ to $(\operatorname{Tr} \rho(\alpha), \operatorname{Tr} \rho(\beta), \operatorname{Tr} \rho(\alpha \beta))$. Then $\mu$ is an embedding of $\widetilde{\mathcal{Q F}}(S)$ into $\left\{(x, y, z) \in \mathbb{C}^{3}: x^{2}+y^{2}+z^{2}=x y z\right\}-\{(0,0,0)\}$ (see for example [1]). Thus we often regard $\widetilde{\mathcal{Q F}}(S)$ as a subset of $\mathbb{C}^{3}$.

For $[\rho] \in \widetilde{\mathcal{Q F}}(S)$, let $x=\operatorname{Tr}(\rho(\alpha)), y=\operatorname{Tr}(\rho(\beta))$ and $z=\operatorname{Tr}(\rho(\alpha \beta))$. In [7], Komori has proved that $\rho \in \mathcal{E}_{\theta_{1}}$ if and only if $\operatorname{Tr}(\rho(\alpha \beta))=\operatorname{Tr}\left(\rho\left(\alpha \beta^{-1}\right)\right)$. On the other hand, Komori and Series have showed in [6] that $\rho \in \mathcal{E}_{\theta_{2}}$ if and only if $\operatorname{Tr}(\rho(\alpha))=\operatorname{Tr}(\rho(\beta))$. We thus have the following theorem.

THEOREM 6.1 ([6], [7]).

$$
\begin{aligned}
& \widetilde{\mathcal{E}}_{\theta_{1}}=\widetilde{\mathcal{Q F}}(S) \cap\left\{(x, y, z) \in \mathbb{C}^{3}: x y=2 z\right\} . \\
& \widetilde{\mathcal{E}}_{\theta_{2}}=\widetilde{\mathcal{Q F}}(S) \cap\left\{(x, y, z) \in \mathbb{C}^{3}: x=y\right\} .
\end{aligned}
$$

Therefore, we have

$$
\widetilde{\mathcal{E}}_{\theta_{1}} \cap \widetilde{\mathcal{E}}_{\theta_{2}}=\{(2 \sqrt{2}, 2 \sqrt{2}, 4),(-2 \sqrt{2},-2 \sqrt{2}, 4)\} .
$$

Note that the two points correspond to the same representation into $\operatorname{PSL}(2, \mathbb{C})$.

Next, we show that

$$
\begin{aligned}
& \widetilde{\mathcal{E}}_{\psi_{1}}=\widetilde{\mathcal{Q F}}(S) \cap\left\{(x, y, z) \in \mathbb{C}^{3}: x=z\right\}, \\
& \widetilde{\mathcal{E}}_{\psi_{2}}=\widetilde{\mathcal{Q F}}(S) \cap\left\{(x, y, z) \in \mathbb{C}^{3}: y=z\right\},
\end{aligned}
$$


where $\psi_{1}, \psi_{2} \in \operatorname{Inv}^{-}(S)_{*}$ are associated with $\left(\begin{array}{cc}1 & 0 \\ 1 & -1\end{array}\right),\left(\begin{array}{cc}-1 & 1 \\ 0 & 1\end{array}\right) \in G L(2, \mathbb{Z})$, respectively (see the proof of Theorem 5.6). In fact, recall that $\theta_{2}$ is an automorphism of $\pi_{1}(S)$ such that $\theta_{2}(\alpha)=\beta$ and $\theta_{2}(\beta)=\alpha$. Let $\tau \in \operatorname{Aut}\left(\pi_{1}(S)\right)$ such that $\tau(\alpha)=\alpha$, and $\tau(\beta)=\alpha \beta$.(Note that the class of $\operatorname{Out}\left(\pi_{1}(\mathrm{~S})\right)$ represented by $\tau$ corresponds to $T=\left(\begin{array}{ll}1 & 1 \\ 0 & 1\end{array}\right)$.) Then $\tau \theta_{2} \tau^{-1} \in \operatorname{Aut}\left(\pi_{1}(S)\right)$ satisfies $\left(\tau \theta_{2} \tau^{-1}\right)(\alpha)=\alpha \beta$ and $\left(\tau \theta_{2} \tau^{-1}\right)(\beta)=\beta^{-1}$. One can see that the class of $\operatorname{Out}\left(\pi_{1}(S)\right)$ represented by $\tau \theta_{2} \tau^{-1}$ corresponds to $\left(\begin{array}{cc}1 & 0 \\ 1 & -1\end{array}\right) \in G L(2, \mathbb{Z})$. Therefore we can write $\psi_{1}=\tau \theta_{2} \tau^{-1}$. For a pair $(\tau(\alpha), \tau(\beta))$ of canonical generators of $\pi_{1}(S)$, we have $\psi_{1}(\tau(\alpha))=\tau(\beta)$ and $\psi_{1}(\tau(\beta))=\tau(\alpha)$. From [6], we know that $[\rho] \in \widetilde{\mathcal{E}}_{\psi_{1}}$ if and only if $\operatorname{Tr}(\rho(\tau(\alpha)))=\operatorname{Tr}(\rho(\tau(\beta)))$, which means $\operatorname{Tr}(\rho(\alpha))=\operatorname{Tr}(\rho(\alpha \beta))$. Thus we obtain the expression of $\widetilde{\mathcal{E}}_{\psi_{1}}$ as above. Similarly, we have the expression of $\widetilde{\mathcal{E}}_{\psi_{2}}$.

A little calculation shows that

$$
\widetilde{\mathcal{E}}_{\theta_{2}} \cap \widetilde{\mathcal{E}}_{\psi_{1}} \cap \widetilde{\mathcal{E}}_{\psi_{2}}=\{(3,3,3)\} .
$$

In the same way, we can obtain

$$
\begin{aligned}
& \widetilde{\mathcal{E}}_{\psi_{3}}=\widetilde{\mathcal{Q F}}(S) \cap\left\{(x, y, z) \in \mathbb{C}^{3}: x y=z+x\right\}, \\
& \widetilde{\mathcal{E}}_{\psi_{4}}=\widetilde{\mathcal{Q F}}(S) \cap\left\{(x, y, z) \in \mathbb{C}^{3}: x y=z+y\right\} .
\end{aligned}
$$

By a calculation, one can see that

$$
\widetilde{\mathcal{E}}_{\theta_{2}} \cap \widetilde{\mathcal{E}}_{\psi_{3}} \cap \widetilde{\mathcal{E}}_{\psi_{4}}=\{(3,3,6)\} .
$$

ACKNOWLEdgment. Grateful acknowledgement is made to my supervisor Professor Kentaro Ito for his considerable help by means of germinal ideas, invaluable guidance and comments. I am also greatly indebted to Professor Yohei Komori and for his valuable comments. I also grateful for the referees' valuable comments and suggestions.

\section{References}

[ 1 ] B. H. Bowditch, Markoff triples and quasi-Fuchsian groups, Proc. London Math. Soc. (3) 77 (1998), no. 3, 697-736.

[ 2 ] C. J. EARLE, Some intrinsic coordinates on Teichmller space, Proc. Amer. Math. Soc. 83 (1981), no. 3, 527531.

[ 3 ] B. FARB and D. Margalit, A primer on mapping class groups, Princeton Mathematical Series 49, Princeton University Press, Princeton, NJ, 2012.

[ 4 ] D. E. FLATH, Introduction to Number Theory, New York, Wiley, 1989.

[ 5 ] L. KeEN, Teichmller spaces of punctured tori: I, II, Complex Variables, Theory and Appl. 2(1983), no. 2, 199-211, 213-225. 
[ 6] Y. Komori, C. Series, Pleating coordinates for the Earle embedding, Ann. Fac. Sci. Toulouse Math. (6) 10 (2001), no. 1, 69-105.

[ 7 ] Y. Komori, A note on a paper of T. Sasaki, Proceedings of the Second ISAAC Congress, Vol. 2 (Fukuoka, 1999), 11091115, Int. Soc. Anal. Appl. Comput., 8, Kluwer Acad. Publ., Dordrecht, 2000.

\section{Present Address:}

Mathematics and Statistical Institute,

HENAN UNIVERSITY,

Jin Ming Avenue, Kaifeng, Henan, 475004, China.

e-mail: mengyifei001@gmail.com 